www.jmscr.igmpublication.org

Impact Factor 3.79

Index Copernicus Value: 5.88

ISSN (e)-2347-176x ISSN (p) 2455-0450

crossref DOI: _http://dx.doi.org/10.18535/jmscr/v4i02.41

\title{
A Study of Predictive Factors Affecting Outcome of IUI in Unexplained Infertility
}

\author{
Authors \\ Dr Namita Shrivastava ${ }^{1}$, Dr Neelesh Shrivastava ${ }^{2}$, Dr Lila Vyas ${ }^{3}$, Dr Anita Sharma ${ }^{4}$ \\ ${ }^{1}$ Senior Resident, SMS Medical College Jaipur Departr, OBS \& GYNAE RUHS \\ Rajasthan University of Health Sciences \\ ${ }^{2}$ Senior Resident, S.S Medical College REWA, (M.P) Avadhesh Pratap Singh University (APSU) \\ ${ }^{3}$ Professor and Unit Head SMS Medical College Jaipur Departr.OBS \& GYNAE .RUHS- \\ Rajasthan University of Health Sciences \\ ${ }^{4}$ Associate Professor, Infertility Unit Head, SSMS Medical College Jaipur Departr. OBS \&GYNAE RUHS- \\ Rajasthan University Of Health Sciences9829564253 \\ Corresponding Author \\ Dr Namita Shriastava \\ Email: namita1.shrivastava@gmail.com
}

\begin{abstract}
Introduction: Infertility is defined as the inability to conceive after 1 year of regular unprotected intercourse, without use of any contraceptive device or methods. Unexplained infertility means that, couple does not conceived after 1 year of unprotected vaginal sexual intercourse, with basic infertility evaluation shows no obvious abnormality (ASRM, 2006) ${ }^{1}$. Incidence $15 \%$ to $30 \%$ of infertile couples (ASRM, 2006) ${ }^{1}$.

Method: 50 cases of unexplained infertility underwent 93 cycles (max. 2 cycles per patient) of IUI both natural as well as stimulated between Feb 2013 -15. In stimulated cycle, COS was initiated and a single IUI performed after 36 hrs of hCG trigger.

Main Outcome Measures: The primary outcome measured was clinical pregnancy rate per cycle and cumulative pregnancy rate. Predictive factors evaluated were age, duration of infertility, type of infertility, stimulation protocol, AFC, ET, endometrial pattern, no. of preovulatory follicles and pre-post wash count and motility.

Results: The pregnancy rate in first and second cycle was $14 \%$ and $20.93 \%$, respectively while cumulative pregnancy rate was $17.20 \%$. Among the predictive factors age 24.69 yrs vs 30.51 yrs $(P=<0.001)$, no. of preovulatory follicles $2.00 \mathrm{vs} 1.56(P=0.046)$, endometrial pattern trilaminar vs diffuse $P R 33.33 \%$ vs $8.12 \%(P$ $=0.04)$ and post wash sperm count $(p<0.001)$ significantly affected the IUI outcome.

Conclusion: Definitive prognostic factors for predicting success will help in counselling regarding treatment modality. Favourable prognostic factors where age, number of preovulatory follicles, motile sperm count and endometrial pattern. The most important factor of these by far is female age, because of its negative effect on ovarian reserve and oocyte quality. By modi modifying the predictive factors significant effect on outcome. When it comes to cost effectiveness, it has been demonstrated that three cycles of IUI offers the same CPR rate as IVF and remain more cost-effective for unexplained infertility. Finally we can say IUI is clearly an efficient treatment option for unexplained infertility.
\end{abstract}

Keywords: Clinical pregnancy rate, intrauterine insemination, predictive factors 


\section{INTRODUCTION}

Infertility is defined as the inability to conceive after 1 year of regular unprotected intercourse, without use of any contraceptive device or methods. Unexplained infertility means that, couple does not conceived after 1 year of unprotected vaginal sexual intercourse, with basic infertility evaluation shows no obvious abnormality (ASRM, 2006) ${ }^{1}$. Incidence $15 \%$ to $30 \%$ of infertile couples (ASRM, 2006) ${ }^{1}$. The Practice Committee of the American Society of Reproductive Medicine (ASRM, 2006) ${ }^{2}$ has published guidelines for standard infertility evaluation: -

1. Confirmation of ovulation history and lab tests.

2. An assessment of fallopian tubes and uterus by HSG.

3. Assessment of semen analysis as per WHO criteria 2010.

If the results of these 3 tests are normal and the couples trying to conceive since 1 year, diagnosis of unexplained infertility criteria made. IUI with or without superovulation is first line treatment choice for unexplained infertility who failed to conceive with ovulation induction and timed intercourse. IUI is the therapeutic process of placing washed spermatozoa transcervically into the uterine cavity and increase the rate of conception by increasing the chance that maximum number of healthy sperms reaches the site of fertilization (ESHRE, 2009). ${ }^{3}$ Proper patient selection, individualization of stimulation protocol and exact timing of insemination, proper technique of sperm preparation are key factors for successful outcome of IUI programme.

\section{METHODS}

The prospective observational study was conducted in Department of Obstetrics and Gynaecology, SMS Medical College, Jaipur from $2013-2014$.
In this prospective study, all 50 couples had atleast one year history of infertility and had undergone standard infertility evaluation and lying in criteria of unexplained infertility. Tubal patency evaluated by hysterosalpinogography. The couple with only one tube patent was included. These couples can undergo a maximum of 2 cycles. Both natural and stimulated cycle were included. Stimulation done with CC (50-150 mg/day) or its combination with gonadotrophins followed by complete monitoring of follicle from D9, once the follicle reached $>17 \mathrm{~mm}$, hCG trigger was given and a single IUI planned after $36 \mathrm{hr}$ later.

On the day of IUI, the husband was instructed to give a semen prepared by swim up technique and density gradient as per patient profile. Both pre and post wash sample parameter assessed. Under all asepsis, IUI carried out with a soft IUI catheter with an insemination volume of $0.5 \mathrm{ml}$. All patients provided luteal support with natural micronized progesterone vaginal pessaries for 16 days. If mensturation was delayed, urine pregnancy test done. Patient not conceived, then underwent second cycle.

Statistical analysis: the variable selected were patient parameter like age of woman, BMI, duration of infertility, type of infertility. Parameters related to ovulation induction like preovulatory follicle number, endometrial thickness and pattern, follicle size at the day of trigger. Lab parameter like preparation of sperm, pre and postwash sperm motility and counts.

\section{RESULTS}

We evaluated 50 patients undergoing a total of 93 cycles, the pregnancy rate in the $1^{\text {st }}$ cycle was $14 \%(7 / 50)$ and in the $2^{\text {nd }}$ cycle was $20.93 \%$. Cumulative pregnancy rate per cycle (16/93) was $17.20 \%$ as shown in Table-1. 
Table - 1_Number of Cycles with Outcome of IUI

\begin{tabular}{lllllll}
\hline \multirow{2}{*}{ Outcome of IUI } & \multicolumn{2}{l}{ Positive } & \multicolumn{3}{l}{ Negative } & Total \\
\cline { 2 - 7 } & No. & $\%$ & No. & $\%$ & No. & $\%$ \\
\hline Cycle1 & 7 & 14.00 & 43 & 86.00 & 50 & 100.00 \\
Cycle2 & 9 & 20.93 & 34 & 79.07 & 43 & 100.00 \\
\hline Total & 16 & 17.20 & 77 & 82.80 & 93 & 100.00 \\
\hline$\chi^{2}=0.369$ & \multicolumn{2}{c}{ d.f. $=1$} & $P=0.544$ & \multicolumn{2}{c}{ NS }
\end{tabular}

The categorical variables were compared using pattern of endometrium, pre and post wash sperm count were significant shown in Table- $2 \& 3$.

Chi-square test. Of all categorical variable age of woman, preovulatory follicle number, trilaminar

Table - 2_Factors Affecting Pregnancy Rates in IUI

\begin{tabular}{|c|c|c|c|c|}
\hline Parameters & $\begin{array}{l}\text { Pregnancies } \\
\text { Cycle }\end{array}$ & / Pregnancy $(\%)$ & Chi-square & P-value \\
\hline \multicolumn{5}{|l|}{ Age Group } \\
\hline $20-25$ & $6 / 23$ & 26.09 & \multirow{4}{*}{18.219} & \multirow{4}{*}{ 0.000, Sig } \\
\hline $26-30$ & $9 / 36$ & 25.00 & & \\
\hline $31-35$ & $1 / 26$ & 3.85 & & \\
\hline $36-40$ & $0 / 8$ & 0.00 & & \\
\hline \multicolumn{5}{|l|}{ BMI } \\
\hline $18.5-29.99$ & $3 / 30$ & 10.00 & \multirow{3}{*}{1.615} & \multirow{3}{*}{$0.446, \mathrm{NS}$} \\
\hline $25-29.99$ & $12 / 58$ & 20.65 & & \\
\hline$>30$ & $1 / 2$ & 20.00 & & \\
\hline \multicolumn{5}{|c|}{ Duration of Infertility } \\
\hline$<5 \mathrm{yrs}$ & $11 / 47$ & 23.91 & \multirow{2}{*}{2.020} & \multirow{2}{*}{$0.155, \mathrm{NS}$} \\
\hline$>5 \mathrm{yrs}$ & $5 / 46$ & 10.64 & & \\
\hline \multicolumn{5}{|c|}{ Case of Infertility } \\
\hline $1^{\mathrm{o}}$ & $12 / 75$ & 16.00 & \multirow{2}{*}{0.079} & \multirow{2}{*}{$0.779, \mathrm{NS}$} \\
\hline $2^{\circ}$ & $4 / 18$ & 22.22 & & \\
\hline \multicolumn{5}{|c|}{ Stimulated Protocol } \\
\hline Natural & $3 / 18$ & 16.67 & \multirow{3}{*}{0.648} & \multirow{3}{*}{$0.723, \mathrm{NS}$} \\
\hline C.C. & $11 / 57$ & 19.30 & & \\
\hline C.C. + FSH & $2 / 18$ & 11.11 & & \\
\hline \multicolumn{5}{|l|}{$\underline{\mathrm{AFC}}$} \\
\hline $0-5$ & $0 / 2$ & 0.00 & \multirow{3}{*}{1.893} & \multirow{3}{*}{$0.355, \mathrm{NS}$} \\
\hline $5-10$ & $2 / 22$ & 9.10 & & \\
\hline $10-15$ & $14 / 69$ & 20.28 & & \\
\hline \multicolumn{5}{|c|}{ Preovulatory Follicle No. } \\
\hline 1 & $4 / 47$ & 8.51 & \multirow{4}{*}{11.678} & \multirow{4}{*}{$0.011, \mathrm{Sig}$} \\
\hline 2 & $6 / 32$ & 18.75 & & \\
\hline 3 & $5 / 12$ & 41.67 & & \\
\hline 4 & $1 / 2$ & 50.00 & & \\
\hline \multicolumn{5}{|c|}{ Endometrial Pallor } \\
\hline Trilaminar & $12 / 36$ & 33.33 & \multirow{3}{*}{11.049} & \multirow{3}{*}{$0.004, \mathrm{Sig}$} \\
\hline \multirow[t]{2}{*}{ Isoechoic } & $4 / 49$ & 8.12 & & \\
\hline & $0 / 8$ & 0.00 & & \\
\hline
\end{tabular}


Table - 3_Factors Affecting The Pregnancy Rate in IUI

\begin{tabular}{llll}
\hline \multirow{2}{*}{ Parameters } & \multicolumn{2}{l}{ Pregnancy } & \multirow{2}{*}{ P-value } \\
\cline { 2 - 3 } & Yes & No & \\
\hline Age & 24.69 & 30.51 & $<0.001, \mathrm{Sig}$ \\
Duration of Infertility & 5.31 & 6.95 & $0.11, \mathrm{NS}$ \\
Mean Follicular Size & 19.75 & 19.64 & $0.889, \mathrm{NS}$ \\
Mean Day of hCG Trigger & 13.22 & 13.69 & $0.25, \mathrm{NS}$ \\
Mean Endometrial Thickness & 8.74 & 8.33 & $0.217, \mathrm{NS}$ \\
Mean Preovulatory Follicle & 2.00 & 1.56 & $0.046, \mathrm{Sig}$ \\
Prewash Sperm Count & 83.81 & 68.84 & $0.02, \mathrm{Sig}$ \\
Prewash Sperm Motility & 65.06 & 67.17 & $0.491, \mathrm{NS}$ \\
Postwash Sperm Count (million/ml) & 49.25 & 33.70 & $<0.001, \mathrm{Sig}$ \\
Postwash Sperm Motility (\%) & 81.56 & 84.42 & $0.365, \mathrm{NS}$ \\
\hline
\end{tabular}

The mean age of patients who had a positive vs negative outcome was 24.69 yrs vs 30.51 yrs $(\mathrm{P}<0.001)$.

Pregnancy rates in patient with duration of infertility $<5$ yr vs $>5$ yr $23.91 \%$ vs $10.64 \%$ ( $\mathrm{P}=0.155)$, was not statistically significant. The mean duration infertility in the positive vs negative outcome group was 5.31 years vs 6.95 years $(\mathrm{P}=0.11)$.

Pregnancy rate in primary infertility vs secondary infertility with PR $16 \%$ vs $22.22 \%$ respectively $(P=0.779)$. Showing type of infertility having no effect on outcome of IUI.

Mean follicular size of dominant follicle in pregnant vs non pregnant patients was 19.75 vs $19.64(\mathrm{P}=0.889)$.

The mean endometrial thickness in patients who had a positive vs negative outcome was $8.74 \mathrm{~mm}$ vs $8.33 \mathrm{~mm}$, $(\mathrm{P}=0.217)$ shows endometrial thickness does not influence IUI outcome.

Highest pregnancy rate of $33.33 \%$ was achieved with trilaminar pattern. Pregnancy rates in trilaminar (12/36 cycles), and isoechoic (diffuse) endometrium (4/49 cycles) were $33.33 \%$ and $8.12 \%$ respectively. None of the patients with irregular endometrium conceived.
The average number of follicles in the positive vs negative outcome group was 2.00 vs $1.56 \quad(\mathrm{P}=0.046)$, shows significantly better results with greater number of follicles prior to IUI. The best pregnancy rate $(58.33 \%)$ observed with 3 preovulatory follicles.

Logistic regression analysis revealed the following predictors age, no. of preovulatory follicles, endometrial pattern remained most significant predictive variable (Table-4) while BMI, duration and type of infertility and endometrial pattern remains non significant. 
Table - 4_Predictors of UPT Positivity in Unexplained Infertility Patients with Binary Logistic Regression

\begin{tabular}{|c|c|c|c|c|c|c|c|}
\hline \multicolumn{2}{|c|}{ Variables in the Equation } & $\mathrm{B}$ & S.E. & Wald & $\mathrm{df}$ & Sig. & $\operatorname{Exp}(B)$ \\
\hline \multirow{8}{*}{ Step $1^{\mathrm{a}}$} & Age & -.677 & .235 & 8.303 & 1 & .004 & .508 \\
\hline & BMI & .287 & .220 & 1.699 & 1 & .192 & 1.332 \\
\hline & Duration & -.168 & .148 & 1.274 & 1 & .259 & .846 \\
\hline & Type of infertility & 1.976 & 1.062 & 3.461 & 1 & .063 & 7.215 \\
\hline & AFC & .201 & .200 & 1.013 & 1 & .314 & 1.223 \\
\hline & Preovulatory Follicle No. & 1.261 & .635 & 3.945 & 1 & .047 & 3.528 \\
\hline & ET & .317 & .369 & .736 & 1 & .391 & 1.373 \\
\hline & Constant & 2.274 & 6.887 & .109 & 1 & .741 & 9.715 \\
\hline
\end{tabular}

a. Variable(s) entered on step 1: AGE, BMI, Duration, type, AFC, Preovulatory Follicle No. the Day of HCG Trigger, ET

\section{DISCUSSION}

In our study, the overall pregnancy rate was $17.20 \%$ in 93 cycles, similar to our study Grigoriou et al (2005) $)^{4}$ and Mervin et al $(2010)^{5}$ reported the pregnancy rate of $14.87 \%$ and $14.7 \%$, high overall pregnancy in our study as all cases were unexplained infertility and it is well known unexplained infertility has best pregnancy rates after IUI all other etiologies of infertility.

We attempt to discover predictive factors for pregnancy after IUI logistic regression analysis carried out 5 predictive variables found out age of women , number of preovlatory follicle, endometrial pattern, pre and post wash sperm count.

The age of the patient significantly affected the pregnancy outcome i.e. younger age was significantly associated with a better pregnancy outcome $(\mathrm{P}<0.001)$. Several studies ${ }^{(6,7,8,9)}$ have shown an association between increasing maternal age and poor pregnancy rates following IUI.

In our study, we found that although the pregnancy rates in patients with $\mathrm{BMI}<25(\mathrm{PR}=$ $10 \%$ ) was lower than in those with $\mathrm{BMI}>25$ $(20 \%)$, however the results were not significant statistically $(\mathrm{P}=0.446)$. The results of our study are supported by Dodson WC et al (2006) ${ }^{10}$, Souter I et al (2011) ${ }^{11}$ and Wolff EF et al (2013) ${ }^{12}$ who also found no significant association between patient's BMI and pregnancy rates following IUI.
In our study increased duration of infertility negatively influences pregnancy rates following IUI. However the results were not significant statistically $(\mathrm{P}=0.11)$. Our findings are supported by several other studies. ${ }^{(6,7,8,9)}$

In our study no significant association present between the type of infertility (Primary or Secondary Infertility) and outcome of IUI ( $\mathrm{P}=$ 0.779). Our findings are consistent with those of Nuojua-Huttunen et al (1999) ${ }^{6}$ achieved a pregnancy rate per cycle of $11.4 \%$ in patients with primary infertility and $14.1 \%$ in patients with secondary infertility following COH-IUI. Infertility type did not significantly affect the outcome of IUI treatment and Kamath MS et al $(2010)^{8}$ found pregnancy rate/cycle of $8.93 \%$ in patients with primary infertility and $12.6 \%$ in patients with secondary infertility. The difference was not significant.

Similar to our study Palatnik A et al $(2012)^{13}$ in their study found higher pregnancy rates were achieved when the leading follicles were in the 23 to $28 \mathrm{~mm}$ range. Although the optimal size of the leading follicle was not statistically significantly different. Azargoon A et al (2013) ${ }^{14}$ found in his study pregnancy rate $36 \%$ with size of follicle (16-19 mm) while $40 \%$ above $20 \mathrm{~mm}$, shows diameter of follicle not significantly associated with IUI outcome.

In our study we found no association between endometrial thickness and IUI outcome. Nuojua- 
Huttunen et al (1999) ${ }^{6}$ found thickness of endometrium was not related with pregnancy outcome and de Araujo LF et al (2013) ${ }^{9}$ in their study not found any difference in pregnancies according to whether endometrial thickness was low or more than $8 \mathrm{~mm}$.

In support to our study most of the studies in literature have obtained significantly better results with greater number of follicles prior to IUI. However most of these studies have used CC or gonadotropins either alone or in combination which promote multi follicular development similar to our study. Nuojua-Huttunen et al $(1999)^{13}$ stated the number of follicles is a predictor of pregnancy rate and found in cycles with single preovulatory follicle the pregnancy rate $(5.7 \%)$ while highest pregnancy rate $(16.3 \%)$ with 3 preovulatory follicles. Asrafi $\mathrm{M}$ et al $(2013)^{8}$ achieved highest pregnancy rate $(22.5 \%)$ with 3 preovulatory follicles.

In our study, a pregnancy rate of $33.33 \%$ with trilaminar pattern while only $8.12 \%$ pregnancy rates with isoechoic (diffuse) pattern $(\mathrm{p}=0.004)$. Our result favoured by Hock DL et al (1997) ${ }^{15}$ who studied on patients receiving sequential $\mathrm{CC}$ and $\mathrm{hMG}$ followed by IUI found that the pregnancy rate was $8 \%$ in the patients with homogenous endometrium determined by TVS performed 15 hours before hCG administration compared to $21 \%$ in patients with a trilaminar pattern on TVS. The difference in PR was significant.

In our study there was significant association of both the prewash sperm count and post wash sperm count while post wash sperm motility is known to have significant association with the IUI results, but in our study tendency does not reached to statistical significance, similarly Asharafi $\mathrm{M}$ et al $(2012)^{8}$ found in their study best pregnancy rates with inseminated motile sperm count $>30 \times 10^{6}$, but the tendency does not reached statistical significance. Azargoon A et al (2013) ${ }^{13}$ in their study found number of motile spermatozoa inseminated having highest impact on pregnancy after IUI treatment.

Definitive prognostic factors for predicting success will help in counselling regarding treatment modality. Favourable prognostic factors where age, umber of preovulatory follicles, motile sperm count and endometrial pattern. The most important of these by far is female age, because of its negative effect on ovarian reserve and oocyte quality. The overall pregnancy rate in our study was $17.20 \%$.

Finally we can say IUI is clearly an efficient treatment option for unexplained infertility.

The actual recommendation is to offer four to six cycles of IUI with or without COS to an unexplained infertility couple and then to shift to IVF, which provides the highest per cycle pregnancy rate $(20-40 \%$ per cycle v/s $9-16 \%$ for IUI) in the shortest interval, but is also the most costly intervention and has high rate of high-order multiple pregnancy. When it comes to cost effectiveness, it has been demonstrated that three cycles of IUI offers the same CPR rate as IVF and remain more cost-effective for unexplained infertility.

\section{REFERENCE}

1. Practice Committee of the American Society for Reproductive. Effectiveness and treatment for unexplained infertility. Medicine Fertil Steril, 2006 Nov; 86(5 Suppl 1) : S111-4.

2. Practice Committee of the American Society for Reproductive Medicine. Optimal evaluation of the infertile female. Fertil Steril, 2006 Nov; 86(5 Suppl 1) : S264-7.

3. ESHRE. Capri workshop. Intrauterine insemination. Hum Reprod Update, 2009; $15: 265-277$.

4. Grigoriou O, Pantos K, Makrakis E, Hassiakos D, Konidaris S, Creatsas G. Impact of isolated teratozoospermia on the outcome of intrauterine insemination. Fertil Steril, 2005; $83:$ 773-775.

5. Merviel P, Heraud MH, Grenier N, Lourdel E, Sanguinet P, Copin H. Predictive factors for pregnancy after 
intrauterine insemination (IUI) : an analysis of 1038 cycles and a review of the literature. Fertil Steril, 2010; 93 : 79-88.

6. Nuojua-Huttunen S, Tomas C, Bloigu R, Tuomivaara L, Markainen H. Intrauterine insemination treatment in subfertility : An analysis of factors affecting outcome. Hum Reprod, 1999; 14 : 698-703.

7. Kamath MS, Bhave P, Aleyamma TK, Nair R, Chandy A. Predictive factors for pregnancy after IUI : A prospective study of factors affecting outcome. Journal of Human Reproductive Sciences, 2010 SepDec; 3(3) : 129-134.

8. Ashrafi M, Rashidi M, Ghasemi A, Arabipoor A, Daghighi S, Pourasghari $\mathrm{P}$, Zolfaghari $\mathrm{Z}$. The role of infertility etiology in success rate of intrauterine insemination cycles: an evaluation of predictive factors for pregnancy rate. New Int J Fertil Steril, 2013 Jul; 7(2) : 100-7.

9. de Araújo LF, de Araújo Filho E, Fácio CL, Bossoni MC, Machado-Paula LA, Corrente JE, Cavagna M, Matheus PC, Pontes A. Efficacy of sperm motility after processing and incubation to predict pregnancy after intrauterine insemination in normospermic individuals. Reprod Biol Endocrinol, 2013 Oct 22; $11: 101$.

10. Dodson WC, Kunselman AR, Legro RS. Association of obesity with treatment outcomes in ovulatory infertile women undergoing superovulation and IUI. Fertility Sterility, Sep 2006; Vol. 86, Issue : pg 642-646.

11. Souter I, Baltagi LM, Kulata DK, Meeker JD, Petrozza JC. Women, weight and fertility : The effect of BMI on the outcome of superovulation / IUI cycles. Fertility Sterility, Mar 2011; Vol. 95, Issue 3 :pg 1042-1047.
12. Wolff EF, Vahidi N, Alford C, Richter $\mathrm{K}$, Widra $\mathrm{E}$. Influences on endometrial development during intrauterine insemination: clinical experience of 2,929 patients with unexplained infertility. Fertil Steril, 2013 Jul; 100(1) : 194-9.e1.

13. Azargoon A, Bahrami M, Alavy Toussy J. Comparing clomiphen citrate plus HMG with clomiphen citrate plus rFSH in IUI cycles in couples with unexplained or male factor infertility: A prospective randomized study. Iran J Reprod Med, 2013 Mar; 11(3) : 243-8.

14. Palatnik A, Strawn E, Szabo A, Robb P. What is the optimal follicular size before triggering ovulation in intrauterine insemination cycles with clomiphene citrate or letrozole? An analysis of 988 cycles. 56. Fertil Steril, 2012 May; 97(5) : 1089-94.e1-3.

15. Hock DL, Bohrer MK, Ananth CV, Kemnnann E. Sonographic assessment of endometrial pattern and thickness in patients treated with $\mathrm{CC}, \mathrm{hMG}$ and IUI. Fertility Sterility, Aug 1997; Vol. 68, Issue $2: \operatorname{pg} 242.1$ 